\section{Perceived depth is a necessary factor in apparent motion concomitant with head motion: A reply to Shebilske and Proffitt}

\author{
WALTER C. GOGEL \\ University of California \\ Santa Barbara, California 93106
}

Shebilske and Proffitt (1981) are critical of the conclusion that the perception of motion of physically stationary objects concomitant with the motion of the head is caused by errors in perceived distance. Instead, they prefer an explanation in terms of the direct perception of retinal motion or perhaps in terms of the minimum principle. Their comments are directed at, not only the experiments reported in "The sensing of retinal motion"' (Gogel, 1980), but also the series of experiments from our laboratory that have used apparent concomitant motion to measure perceived distance and to distinguish between cognitive and perceptual factors in distance responses. This reply will discuss the evidence for and the logic of implicating depth perception, first as an important and then as a necessary variable in the apparent motion of stationary objects concomitant with the motion of the head. It will be shown that the role of perceived distance in this phenomenon cannot be avoided by dismissing apparent concomitant motion as paradoxical, or as determined by retinal motion alone, nor can it be explained, using the minimum principle, as a derivative of retinal motion. The criticisms of the study by Gogel (1980) with regard to procedure and with regard to its implication for the cue of motion parallax will be shown to be inappropriate. Finally, the application of apparent concomitant motion to the measurement of perceived distance and to the separation of cognitive and perceptual factors will be considered briefly.

\section{Basic Evidence}

The evidence that errors in perceived distance are important in producing apparent concomitant motions is unequivocal. Hay and Sawyer (1969) modified the apparent egocentric distance to an object by changing the convergence of the eyes to the object by using Polaroid filters. Apparent concomitant motion occurred for a nodding motion of the head and was measured by physically moving the object in an opposite direction to cancel the apparent motion. Similarly, Wallach, Yablick, and Smith (1972) changed apparent distance by varying both accommodation and convergence, with the resulting apparent con-

The preparation of this paper was supported by Research Grant BNS 77-16620 from the National Science Foundation. comitant motion again measured by the canceling procedure. In order to explain these results in terms of retinal motions, one would need to postulate large errors in the direction of gaze that vary systematically with a change in the position of the head, with this pattern of retinal motions varying as a function of distance cues. However, all hope of explaining the apparent motions of stationary objects concomitant with a motion of the head without reference to apparent distance disappears when experiments or demonstrations involving an illusory depth orientation of extended objects or parts are considered. The reason for this is that the retinal motion between physically stationary objects or parts of a scene (relative retinal motion) is independent of eye movements or conditions of eye fixation. Instead, for a given amount of head motion, relative retinal motion depends only on the physical (not the apparent) distances of the portions of the scene from the observer. Thus, an object oriented vertically in a frontoparallel plane does not rotate clockwise or counterclockwise on the retina as the head is moved laterally. Nevertheless, perceived concomitant rotation can occur under this condition, as is shown by a study by Gogel and Tietz (1974) in which the perceived slant of a trapezoidal figure was varied relative to its physical slant by means of perspective size cues. When the object was physically in a frontoparallel plane, but apparently slanted in depth, it appeared to rotate in the plane of its apparent depth slant as the head was moved, with the direction of rotation determined by the direction of the apparent slant. The reader can experience a similar effect by viewing a line stereogram or random dot stereogram physically produced on a flat page but extending perceptually upward toward the face. In this case, a lateral motion of the head will cause the scene to sway (rotate) in apparent depth with the apparently near portions of the scene, relative to the far portions, appearing to move in the same direction as the head motion. Of course, since all parts of the scene are physically nearly at the same distance from the observer, very little relative motion is present on the eyes as the head is moved.

In the study by Gogel and Tietz (1974), as in the study entitled "The sensing of retinal motion"' (Gogel, 1980), the extended object was sometimes slanted in physical depth, with cues of illusory depth (perspective and binocular disparity in the two studies, respectively) producing an error in the perceived slant of the object in depth. In these cases, concomitant rotation on the eye was present and, again, the illusory depth cues produced a predictable variation in perceived rotation. Common examples of this phenomenon are the perception of apparent turning in depth of a stationary (inverted) face mask or of a three-dimensional Necker 
cube when the mask or cube is seen as reversed in depth and is viewed with a moving head. In all these instances, including the study of "The sensing of retinal motion" (Gogel, 1980), perceived concomitant motion varied with changes in perceived depth (for a constant retinal motion). Clearly, such results cannot be explained in terms of retinal motion alone. Are there any alternatives to accepting apparent depth as an important factor in perceived concomitant motion? Shebilske and Proffitt (1981) state that in the study by Gogel (1980) "the motions reported could also have been based solely on motion information and principles of perceptual organization that make no use of distance information." They further go on to say, "Under the assumption that the minimum principle is primary, one might argue that distance information was a derivative of motion information in Gogel's experiment." These statements are based on a misunderstanding. In the study by Gogel, three physical slants in depth of a line $20 \mathrm{deg}$ top far, vertical, and $20 \mathrm{deg}$ top near were used. At each of these physical slants, five different stereoscopic slants were superposed using a Polaroid stereoscope so that systematic variations occurred in the perceived depth slant of the line. The physical rotation on the eye as the head was moved was determined solely by the physical slant, not by the added stereoscopic slant. Thus, with a particular physical slant, say 20 deg top far, the rotation on the retina remained constant, whereas the perceived slant varied as a function of the stereoscopic slant added by the stereoscope. It follows, for a constant physical slant, that no changes in the rotation on the eye were available to be used together with the minimum principle to produce either the changes in perceived slant or the changes in perceived rotation, or the high correlations obtained in this study between these two variables.

\section{The Equidistance Tendency and Apparent \\ Concomitant Motion}

When viewing objects at different physical distances with the head moving, apparent concomitant motion between the objects often will occur, with the directions of the motions consistent with and explainable by a perceptual underestimation of the depth. But why should depth usually be underestimated? An answer is found in the equidistance tendency, which often will produce a reduction in apparent depth even under conditions in which some depth cues are available, with the amount of underestimation increasing with increased cue reduction (Gogel, 1965). An instance of apparent concomitant motion due to the equidistance tendency is the apparent relative motion between two objects (e.g., two dowels) positioned at different physical distances and viewed with the head moving. To find out whether such apparent motions are caused by errors in perceived depth, as expected from the equidistance tendency, an effect found in a study by Wist and Summons (1976) was applied. According to Wist and Summons, the tendency to perceive one object at the distance of another increases with fixation on the latter object. Thus, objects farther and nearer than the fixated object will be displaced perceptually toward the distance of fixation, and apparent concomitant motions should occur in directions predicted from the resulting errors in perceived distance. To investigate this possibility, the apparent concomitant motion of a luminous point suspended in a visual alley was measured as a function of whether the fixation object was at a distance closer or farther than the point. Apparent concomitant motions of the point were obtained in the directions predicted from the equidistance effects (Gogel, 1979; Gogel \& Tietz, 1977), with the magnitude of the apparent concomitant motion measured either by combining it vectorially with the apparent motion from a physical vertical motion or by canceling it with a physical motion in the opposite direction. The change in perceived distance in the predicted direction also was found using a stationary head and measuring perceived distance by its effect on apparent size.

\section{Are Apparent Concomitant Motions Paradoxical?}

The direction, but often not the magnitude, of the apparent concomitant motions that occur between objects because of the perceptual underestimation of depth is consistent with the relative motions of the images of these objects on the eye. Despite the evidence discussed above, Shebilske and Proffitt interpret these apparent concomitant motions in terms of the direct perception of relative retinal motion. Seemingly in support of this interpretation they assert that apparent concomitant motions are paradoxical, that is, the objects are seen in the same spatial location before and after the head is moved. As evidence for this conclusion, they state "one can point to them accurately before and after one's head moves, even though one sees apparent relative motion during the head movement." Unfortunately, the expression "point to" is ambiguous. If the pointing is to the direction, not to the distance, of the object, accuracy is expected from the point of view expressed in this paper. As has been discussed elsewhere (for example, see Gogel \& Tietz, 1974), an explanation of apparent concomitant motion in terms of errors in depth perception requires the ability to perceive direction with at least reasonable accuracy. We assert that it is the accuracy of perceived direction combined with an inaccuracy of perceived relative distance that is responsible for the perception of the relative motion of stationary objects concomitant with movement of the head.

Also, experimental evidence is available to conclude that apparent concomitant motion is subjectively the same as apparent motion resulting from viewing 
a physically moving object (except, of course, that the former apparent movement is concomitant with the motion of the head). As noted in the above discussion, real motion can combine vectorially with or can cancel apparent concomitant motion. Finally, as shown in a recent study (Gogel \& Tietz, 1979), the observer can adjust a physical motion to perceptually duplicate an apparent concomitant motion of a physically stationary object, with this adjustment consistent with the results obtained from a vectorial procedure. On the basis of this evidence, it is difficult to escape the conclusion that apparent concomitant motions produced by errors in perceived distance and apparent motions produced in more standard ways are subjectively equivalent.

\section{Perception of Depth as a Necessary Condition for Apparent Concomitant Motion}

Clearly, apparent depth is a ubiquitous factor in apparent concomitant motion. It can be shown by an extension of the previous discussion that it is also a necessary factor. Consider Figure 1, which illustrates a situation in which the perceived $\left(\mathrm{e}^{\prime} \mathrm{f}^{\prime}\right)$ and physical (ef) depths between two points are reversed. For example, $e$ and $f$ could be physically near and far points on a perceptually inverted Necker cube or face mask. As illustrated, consistent with the assumption of the veridicality of perceived direction, the perceived direction to each point is defined by its physical direction from the observer. Again, the retinal motion is determined by the physical depth (for a given motion of the head). Thus, for a constant physical depth, the relation between retinal and apparent concomitant motion (or rotation $\beta^{\prime}$ ) can be varied by varying the apparent depth positions $\mathrm{e}^{\prime}$ and $\mathrm{f}^{\prime}$ along the direction lines to the points, as shown in Figure 1. If $\mathrm{e}^{\prime}$ and $f^{\prime}$ are at $e$ and $f$, no apparent motion will occur $\left(\beta^{\prime}=0\right)$; if the perceived depth $e^{\prime} f^{\prime}$ is less than or is opposite to the physical depth ef, apparent and retinal concomitant rotation will be in the same direction. But even in this latter case, the magnitude of $\beta^{\prime}$ will be consistent with the retinal motions if and only if $\mathrm{e}$ and $\mathrm{f}$ appear in the same frontoparallel plane $\left(\beta^{\prime}=180 \mathrm{deg}\right)$. It follows that apparent depth (including apparent equidistance) as well as retinal rotation must be considered under all conditions if $\beta^{\prime}$ (including the cases in which $\beta^{\prime}=180$ and $0 \mathrm{deg}$ ) is to be predicted. According to this analysis, apparent depth or distance must always be considered in the perception of apparent concomitant motion.

It follows from Figure 1 that whenever the perceived depth between $e$ and $f$ is greater than the physical depth, apparent concomitant rotation opposite to retinal rotation will occur. This is the prediction tested in "The sensing of retinal motion" (Gogel, 1980). The main procedural objections of Shebilske and Proffitt to this study are: (1) Direct measures of

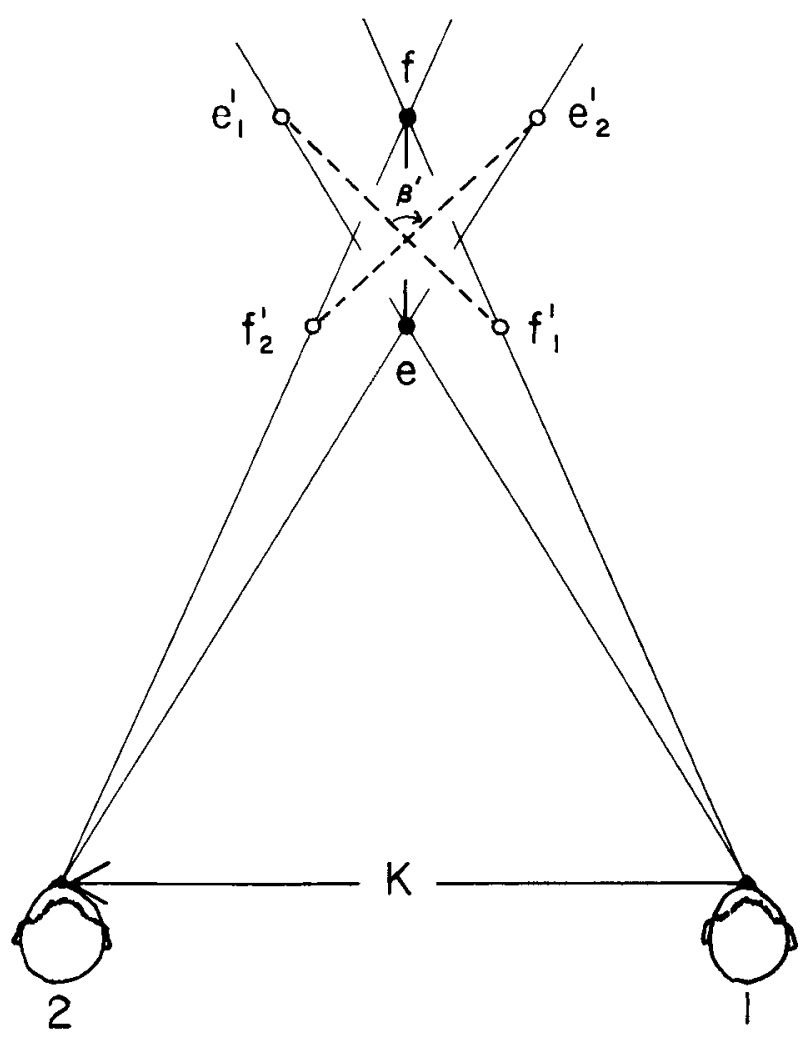

Figure 1. Apparent rotation, $\beta^{\prime}$, between two points (ef) as a function of the perceived orientation ( $\left(e^{\prime} f^{\prime}\right)$ of the points in depth, as viewed with a laterally moving head.

the depth between the ends of the line were not obtained; (2) the perception of the egocentric distance of the center of the line was not measured; (3) the perceptions at the extreme positions of the head may not have been representative of the apparent motions throughout the extent of head motion; and (4) telling the observers to ignore the motions of the ends of the lines in judging the angle of apparent rotation in Experiment 2 might have caused them to attend only to the extreme apparent positions of the line. These four procedural concerns can be resolved and are addressed in the same order below.

(1) Equation 3 (Gogel, 1980, p. 157) specifies the factors that must be measured in that study in order to predict the perceived rotation $\left(\beta^{\prime}\right)$. These include the physical $(\phi)$ and perceived $\left(\phi^{\prime}\right)$ slope of the line in depth. A measure of the perceived distance $\left(D^{\prime}\right)$ of each end of the line is not required. As shown by Equation 2 (p. 156), measures of the perceived distances of the ends of the line are required only if the apparent concomitant motion ( $\left.W^{\prime}\right)$ of each end of the line is to be predicted. (2) Despite the fact that the convergence distance to the center of the stimulus line was always constant at $1 \mathrm{~m}$, it is likely from the specific distance tendency (Gogel, 1969) that the 
center of the line was perceived usually to be more distant than $1 \mathrm{~m}$. But it was unnecessary to measure this egocentric error in perceived distance because this error adds the same component of apparent concomitant motion (apparent translation) to all parts of the line (see Gogel \& Tietz, 1974), with the perceived rotation $\left(\beta^{\prime}\right)$ remaining unaffected. Thus, measures of neither apparent egocentric distance nor the apparent translatory motion resulting from likely errors in perceived egocentric distance were necessary for the study. (3) The fact that it has been shown previously that apparent concomitant motion can be canceled by, and can combine vectorially with, a simple physical motion indicates, in this study also, that the path of the apparent concomitant motion must have been simple throughout the head motion. (4) The apparent movement of the ends of the stimulus line was determined by the apparent length of the line as well as by its apparent angle of rotation. For this reason, it was desirable to instruct the observer to ignore the former variable in judging the angle of apparent rotation. Although the same conclusions were supported by Experiments 1 and 2, the added instructions were useful, as is indicated by the increase in similarity of the predicted and obtained data in Experiment 2.

The basic design of the study by Gogel (1980) is simply that suggested by Figure 1. The question of whether apparent concomitant rotation can occur opposite to that expected from retinal rotation alone was investigated by increasing the stereoscopic depth separation within the display, with the physical depth separation held constant. When the stereoscopic separation in the same direction as the physical separation was sufficiently large, the predicted result occurred. In addition, it was found that the function relating perceived depth (slant) and perceived rotation was continuous (see Figures 4 and 6 of that study), even when the retinal and perceptual rotations were opposite in direction. It is concluded that both retinal rotation and perceived slant are essential to the perception of motion concomitant with a motion of the head and that the function describing the interaction of these two factors is the same regardless of whether these factors are in the same or opposite directions.

\section{Motion Parallax and Apparent Concomitant Motion}

The study by Gogel (1980) does not discuss motion parallax directly, but it has implications for this concept. In the opening sentence of their comments, Shebilske and Proffitt (1981) state that "Gogel (1980) argued from two experiments that motion parallax is a result of perceptual underestimation of depth between objects." This statement is incorrect. In the Gogel article, apparent concomitant motion and motion parallax are not equated. Shebilske and
Proffitt, on the other hand, do equate these phenomena, since, in the next paragraph, they state, "Motion parallax is a set of apparent motions of stationary objects that results from a change in viewing position." A conclusion consistent with the Gogel (1980) article is that the perception of motion of physically stationary objects at different physical distances, concomitant with head motion, represents a failure of (not a definition of) the motion parallax cue of distance. If the motion parallax had been completely effective, the objects would have appeared to be stationary and would have been perceived to be at their physical distances. To suggest that they both appear to move and are perceived correctly in distance is like having one's cake and eating it too! Rather than defining the motion parallax cue of relative distance from head motion in terms of apparent motions, it ought to be defined as the relative motions of images on the retina per unit of head motion. Shebilske and Proffitt go on to state that "Gogel's attempt to tie retinal motion hypotheses to the more general corecontext theory is misleading." It is misleading, they suggest, because this would require that motion parallax varies with distance information. As discussed in relation to Figure 1, if distance cues are reduced, objects will tend to appear equidistant and their apparent concomitant motions indeed will be consistent with their retinal motions in both magnitude and direction. But motion parallax would change in this manner with cue reduction only if it were defined in terms of apparent motions. It is Shebilske and Proffitt's unfortunate choice of a definition that leads them to this dilemma.

\section{Applications of the Head Motion Procedure}

The validity of the head motion procedure in measuring apparent distance depends upon the validity of the interpretation of apparent concomitant motion presented in this paper. The assumption basic to all phenomena of apparent concomitant motion is that during a head motion the observer can correctly perceive the change in the direction of objects per unit of sensed motion of the head. If this occurs with a reasonable degree of accuracy, the head-motion procedure can be expected to measure apparent distance, and with this same accuracy. Substantial systematic errors in this perceived ratio, however, will reduce, but not necessarily eliminate, the usefulness of this procedure for measuring apparent distance. The evidence is firm that perceived distance, as measured with the head motion procedure using a canceling (null adjustment) technique is a monotonically increasing function of the distance actually perceived. Whether it is a linear function of, or is proportional to, perceived distance remains to be determined. The headmotion procedure has been applied, with consistent and meaningful results, to the measurement of per- 
ceived depth from convergence, accommodation, and absolute motion parallax (Gogel, 1977; Gogel \& Tietz, 1979), to the measurement of perceived distance from the familiar and relative size cues of distance (Gogel, 1976), and to the measurement of the distance indicated by the specific distance tendency (Gogel \& Tietz, 1977).

An important advantage that the head-motion procedure has over some other, more direct, methods of measuring perceived distance is that the relation between apparent motion and apparent distance is not obvious to the observer (and, as it seems from this exchange, sometimes not to other researchers as well). Thus, the observer is unlikely to modify his or her adjustment, using this procedure, in an effort to be veridical. It follows that the measurement of perceived distance with the head-motion procedure is likely to be free from nonperceptual (cognitive) effects that sometimes occur, for example, with verbal reports of distance, and probably also with reaching to the distance of the object. The head-motion procedure has been used in our laboratory to distinguish between cognitive and perceptual factors in phenomena such as the familiar size cue of distance (Gogel, 1976) and the effect of posthypnotic suggestion on perceived distance (MacCracken, Gogel, \& Blum, 1980).

Shebilske and Proffitt end their comments with the statement that the interpretation provided by our research concerning the relation between perceived motion and perceived distance is "at odds with other current theories of motion perception." This criticism, like those directed at the distinction between cognitive and perceptual factors in spatial responses, is too vague to permit a detailed response. Certainly there is evidence for the involvement of perceived distance in other aspects of motion perception, such as motion constancy (Rock, Hill, \& Fineman, 1968). More generally, perceived distance is involved in a number of perceptions studied in a variety of constancy phenomena. The interrelation of apparent distance and apparent concomitant motion is a special case of a more general concept that we have described as perceptual interactions or perceptual equations (Gogel, 1973). This concept has some similarities to what has been termed the "algorithm" or "taking-into-account" approach to perception (Ebenholtz, 1977; Epstein, 1973; Rock, 1977). For example, in the "sensing" study (Gogel, 1980), the only distal event that is consistent with the apparent depth slant and apparent rotation of the stimulus line opposite to the retinal rotation is a line physically slanted and moving in the manner perceived. Although the point of view expressed in this paper is more analytical and less experiential than this, it follows that the assertion of the role of apparent depth in apparent concomitant motion is not inconsistent with all current theory.

In summary, before the equidistance and specific distance tendencies were identified, the idea that apparent depth was unavoidably involved in apparent concomitant motion could be denied on the basis that apparent depth was not available under conditions of cue reduction. Considering these tendencies, however, and particularly in view of the results obtained in the "sensing" and other studies, the conclusion that perceived distance is always involved in the apparent concomitant motion of physically stationary objects is increasingly credible.

\section{REFERENCES}

Ebenholtz, S. The constancies in object orientation: An algorithm processing approach. In W. Epstein (Ed.), Stability and constancy in visual perception: Mechanisms and processes. New York: Wiley, 1977.

EPSTE in, W. The process of 'taking-into-account' in visual perception. Perception, 1973, 2, 267-285.

Gogel, W. C. Equidistance tendency and its consequences. Psychological Bulletin, 1965, 64, 153-163.

Goget, W. C. The sensing of retinal size. Vision Research, 1969, 9, 3-24.

Gogel, W. C. The organization of perceived space I: Perceptual interactions. Psychologische Forschung, 1973, 36, 195-221.

GoGEL, W. C. An indirect method of measuring perceived distance from familiar size. Perception \& Psychophysics, 1976, 20, 419-429.

Gogel, W. C. An indirect measure of perceived distance from oculomotor cues. Perception \& Psychophysics, 1977, 21, 3-11.

GoGEL, W. C. The common occurrence of errors of perceived distance. Perception \& Psychophysics, 1979, 25, 2-11.

Gogel, W. C. The sensing of retinal motion. Perception \& Psychophysics, 1980, 28, 155-163.

Gogel, W. C., \& TIETz, J. D. Absolute motion parallax and the specific distance tendency. Perception \& Psychophysics, 1973, 13, 284-292.

Gogel, W. C., \& TiETZ, J. D. The effect of perceived distance on perceived movement. Perception \& Psychophysics, 1974, 16, 70-78.

Gogel, W. C., \& Tietz, J. D. Eye fixation and attention as modifiers of perceived distance. Perceptual \& Motor Skills, 1977, 45, 343-362.

Gogel, W. C., \& Tietz, J. D. A comparison of oculomotor and motion parallax cues of egocentric distance. Vision Research, $1979,19,1161-1170$.

Hay, J. D., \& SaWyer, S. Position constancy and binocular convergence. Perception \& Psychophysics, 1969, 5, 310-312.

MacCracken, P. J., Gogel, W. C., \& Blum, G. S. Effects of post-hypnotic suggestion on perceived egocentric distance. Perception, 1980, 9, 561-568.

Rock, I. In defense of unconscious inference. In W. Epstein (Ed.), Stability and constancy in visual perception: Mechanisms and processes. New York: Wiley, 1977.

Rock, I., Hill, A. L., \& Fineman, M. Speed constancy as a function of size constancy. Perception \& Psychophysics, 1968, 4, 425-440.

Shebilske, W. L., \& Proffitt, D. R. The priority of perceived distance for perceiving motion has not been demonstrated: Critical comments on Gogel's "The sensing of retinal motion." Perception \& Psychophysics, 1981, 29, 170-172.

Wallach, H., Yablick, G., \& Smith, A. Target distance and adaptation in distance perception in the constancy of visual direction. Perception \& Psychophysics, 1972, 12, 139-145.

Wist, E. R., \& Summons, E. Spatial and fixation conditions affecting the temporal course of change in perceived relative distance. Psychological Research, 1976, 39, 99-112.

(Received for publication November 12, 1980; accepted December 29, 1980.) 